\title{
About the Closed Quasi Injective S-Acts Over Monoids
}

\author{
Shaymaa Amer Abdul-Kareem ${ }^{1,}$, Ahmed Amer Abdulkareem ${ }^{2}$ \\ ${ }^{1}$ Department of Mathematics, College of Basic Education, Mustansiriyah University, Baghdad, Iraq \\ ${ }^{2}$ Department of Computer Science, College of Science, Mustansiriyah University, Baghdad, Iraq
}

Email address:

Shaymma_amer.edbs@uomustansiriyah.edu.iq (S. A. Abdul-Kareem), vip_amer46@yahoo.com (A. A. Abdulkareem)

${ }^{*}$ Corresponding author

\section{To cite this article:}

Shaymaa Amer Abdul-Kareem, Ahmed Amer Abdulkareem. About the Closed Quasi Injective S-Acts Over Monoids. Pure and Applied Mathematics Journal. Special Issue: Algebra with Its Applications. Vol. 8, No. 5, 2019, pp. 88-92.

doi: 10.11648/j.pamj.20190805.12

Received: September 8, 2019; Accepted: October 15, 2019; Published: October 24, 2019

\begin{abstract}
The aim of introducing and studying the notion of closed quasi injective S-act is to create a basis facilitate for the exchange ideas between module theory and act theory. As well as it represents a generalization of the quasi-injective act. The quasi-injective act was first introduced and studied by A. M. Lopez, Jr. and J. K. Luedeman, 1979. Then the author was one of the researchers which introduced several generalizations for this notion from several aspects because of its importance. More accurately, the contribution of this paper to the field of competence can be summarized into three points as follows: First: The possibilities for applying the topic of this article helps researchers about how can connect class of injectivity with its generalizations. Second: Study the topic of this article contributes to the improvement of the vision for finding the corresponding between acts theory and module theory. Third: This article has dealt with the important subject in the field of science and knowledge especially in algebra and can take it as a basis for future work for the researchers who work on algebra. Now, in this paper, the concept of closed quasi injective acts over monoids is introduced which represents a generalization of quasi injective. Several characterizations of this concept are given to show the behavior of the property of closed quasi injective. Relationship of the concept of closed quasi injective acts over monoids with Hopfian, co-Hopfian and directly finite property are considered. This work gives the answer to the question of what are the conditions to be met in the subacts in order to inherit the property of closed quasi injectivity. We obtained the main result in this direction in proposition (2.5) and proposition (2.6). A part of this paper was devoted to studying the relationship among the class of closed quasi injective acts with some generalizations of injectivity.
\end{abstract}

Keywords: Closed Quasi Injective Acts, Extending Acts, Continuous Acts, Noetherian Acts, Hopfian Acts

\section{Introduction}

Actions of a semigroup have always been interesting to mathematicians. From an algebraic perspective, a semigroup action is a generalization of the notion of group action in group theory. Besides, it is familiar that in the theoretical computer science and in algebra, an action of a semigroup on a set is a rule which associates to each element of the semigroup is a transformation of the set in such a way that the product of two elements of the semigroup is associated with composite of two corresponding transformations. The terminology conveys the idea that the elements of the semigroup are acting as transformations of the set. An important special case is a monoid action or act, in which the semigroup is a monoid and the identity element of the monoid acts as the identity transformation of a set. It is recognized that the theory of monoids and systems is a generalization of the theory of rings and modules, which has a number of direct applications in theoretic Computer science, Theory of differential equations and Functional analysis, etc.

Now, by a monoid $\mathrm{S}$ we always mean monoid with zero elements 0 and every right $\mathrm{S}$-act $\mathrm{M}$ is unitary with zero element $\Theta$ which denoted by $M_{S}$. A right $S$-act $M_{S}$ with zero is a non-empty set with a function $\mathrm{f}: \mathrm{M} \times \mathrm{S} \rightarrow \mathrm{M}, \mathrm{f}(\mathrm{m}, \mathrm{s}) \mapsto$ ms such that the following properties hold: (1) $\mathrm{m} \cdot 1=\mathrm{m}$ (2) $\mathrm{m}$ $(s t)=(m s) t$, for all $m \in M$ and $s, t \in S, 1$ is the identity element of $\mathrm{S}$. It is possible to find the S-act with several names as follows: S-systems, S-sets, S-operands, S-polygons, 
Transition systems, S-automata [1]. Note that we will use terminology and notations from [2-5] freely. For more details about injective acts and their generalizations we refer the reader to the references [6-8]. Familiar concepts are good and natural stations for starting. Recall that a non-zero subact $\mathrm{N}$ of $M_{S}$ is intersection large if for all non-zero subact $A$ of $M_{S}$, $A \cap \mathrm{N} \neq \Theta$, and will be denoted by $\mathrm{N}$ is $\cap$-large in $\mathrm{M}_{\mathrm{S}}$. Equivalently, if for each $\Theta \neq m \in M_{S}$ there exists $s \in S$ such that $\Theta \neq m s \in N$ [9]. In this case, we call $M_{S}$ is $\cap$-large extension of N. An S-homomorphism f which maps an S-act $\mathrm{M}_{\mathrm{S}}$ into an S-act $N_{S}$ is said to be split if there exists S-homomorphism $g$ which maps $N_{S}$ into $M_{S}$ such that fog $=1_{M}[10]$.

Let $A_{S}, M_{S}$ are two $S$-acts. $A_{S}$ is called $M$-injective if given an S-monomorphism $\alpha: \mathrm{N} \rightarrow \mathrm{M}_{\mathrm{S}}$ where $\mathrm{N}$ is a subact of $\mathrm{M}_{\mathrm{S}}$ and every S-homomorphism $\beta: \mathrm{N} \rightarrow \mathrm{A}_{\mathrm{S}}$, can be extended to an S-homomorphism $\sigma: M_{S} \rightarrow A_{S}[11]$. An $S$-act $A_{S}$ is injective if and only if it is M-injective for all S-acts $M_{S}$. An S-act $A_{S}$ is quasi injective if and only if it is A-injective. Quasi injective S-acts have been studied by Lopez and Luedeman [12]. A subact $\mathrm{N}$ of $\mathrm{S}$-act $\mathrm{M}_{\mathrm{S}}$ is called closed if it has no proper $\cap$ large extension in $\mathrm{M}_{\mathrm{S}}$ that is the only solution of $\mathrm{N} \hookrightarrow^{n l} \mathrm{~L}$ $\neq \hookrightarrow \mathrm{M}_{\mathrm{S}}$ is $\mathrm{N}=\mathrm{L}$ [13]. An $\mathrm{S}$-act $\mathrm{M}_{\mathrm{S}}$ is said to satisfy $\mathrm{C}_{1}$ condition if every closed subact of $\mathrm{M}_{\mathrm{S}}$ is a retract subact of $M_{S}$. An S-act $M_{S}$ is said to satisfy $C_{2}$-condition if every subact of $M_{S}$ which is isomorphic to retract subact of $M_{S}$ is itself a retract subact of $\mathrm{M}_{\mathrm{S}}$. An S-act $\mathrm{M}_{\mathrm{S}}$ is called a CS-act or extending act if it is satisfied $\mathrm{C}_{1}$-condition. An $\mathrm{S}$-act $\mathrm{M}_{\mathrm{S}}$ is called continuous if it is satisfied $C_{1}$ and $C_{2}$-conditions [14]. In early time we gave some generalizations of the quasiinjective S-acts, which represent generalizations of the doctoral dissertation of the author. Now, we adopt another generalization of quasi injective act which is C-quasi injective acts to characterize the behavior of the property considered under well-known constructions such as product, coproduct, and direct sum.

This article consists of three sections. The first one (section two) is devoted to introduce and investigate a new kind of generalization of quasi-injective S-acts, namely Cquasi injective over monoids. Certain classes of subacts which inherit the property of C-quasi injective acts were considered. Also, the characterizations of this new class of Sacts were investigated. An example was given to demonstrate $\mathrm{C}$-quasi injective acts over monoids. Some known results on $\mathrm{C}$-quasi injective for general modules were generalized to Sacts. In the second part (section three) has clarified the discussion for our results. The third part (section four) has clarified the conclusions of our work.

\section{Main Results}

Definition (2.1): Let $\mathrm{M}_{\mathrm{S}}$ and $\mathrm{N}_{\mathrm{S}}$ be two $\mathrm{S}$-acts, $\mathrm{N}_{\mathrm{S}}$ is called closed M-injective (for short $\mathrm{C}$-M-injective) if for any homomorphism from a closed subact of $\mathrm{M}_{\mathrm{S}}$ to $\mathrm{N}_{\mathrm{S}}$ can be extended to homomorphism from $\mathrm{M}_{\mathrm{S}}$ to $\mathrm{N}_{\mathrm{S}}$. An S-act $\mathrm{N}_{\mathrm{S}}$ is called closed quasi injective if $\mathrm{N}_{\mathrm{S}}$ is $\mathrm{C}-\mathrm{N}$-injective. A monoid $\mathrm{S}$ is called right closed self-injective if it is $\mathrm{C}$-S-injective.

Remark and Example (2.2):

(1) Every quasi injective act is closed quasi injective (simply C-quasi injective), but the converse is not true in general, for example $\mathrm{Z}$ with usual multiplication is $\mathrm{C}$-quasi injective $Z$-act, but it is not quasi injective.

(2) Obviously, definition (2.1) is up to isomorphism. This means that isomorphic act to $\mathrm{C}$-quasi injective act is $\mathrm{C}$-quasi injective.

Lemma (2.3): Let $\mathrm{A}_{\mathrm{S}}$ and $\mathrm{B}_{\mathrm{S}}$ are two $\mathrm{S}$-acts. If $\mathrm{f}: \mathrm{A}_{\mathrm{S}} \rightarrow \mathrm{B}_{\mathrm{S}}$ is isomorphism and $X$ is $\cap$-large subact of $A_{S}$, then $f(X)$ is $\cap$ large subact of $\mathrm{B}_{\mathrm{S}}$.

Proof: For each $\mathrm{f}(\mathrm{Y})$ which is any non-zero subact of $\mathrm{B}_{\mathrm{S}}$ there exists a non-zero subact $Y$ of $A$ (Since $f$ is isomorphism). As $\mathrm{X}$ is $\mathrm{\cap}$-large subact of $\mathrm{A}$, so we have $\mathrm{X} \cap \mathrm{Y} \neq \Theta$. Now, we must prove that $\mathrm{f}(\mathrm{X}) \cap \mathrm{f}(\mathrm{Y}) \neq \Theta$. Since $\mathrm{f}$ is monomorphism, so $\mathrm{X} \cap \mathrm{Y} \subseteq \mathrm{f}(\mathrm{X}) \cap \mathrm{f}(\mathrm{Y})$, but $\mathrm{X} \cap \mathrm{Y} \neq \Theta$. Hence $\mathrm{f}(\mathrm{X}) \cap \mathrm{f}(\mathrm{Y}) \neq \Theta$ and $\mathrm{f}(\mathrm{X})$ is $\cap$-large subact of $\mathrm{B}$.

Proposition (2.4): Let $\mathrm{N}$ is a closed subact of $\mathrm{S}$-act $\mathrm{M}_{\mathrm{S}}$. If $\mathrm{N}$ is C-M-injective act, then any monomorphism from $\mathrm{N}$ into $\mathrm{M}_{\mathrm{S}}$ split (in other words if $\mathrm{N}$ is $\mathrm{C}-\mathrm{M}$-injective act, then $\mathrm{N}$ is a retract subact of $\mathrm{M}_{\mathrm{S}}$ ).

Proof: Let $\alpha: \mathrm{N} \rightarrow \mathrm{M}_{\mathrm{S}}$ be monomorphism such that $\mathrm{N}$ is closed subact of $\mathrm{M}_{\mathrm{S}}$. Since $\mathrm{N}$ is C-M-injective, so there exists S-homomorphism $\beta: M_{S} \rightarrow N$ such that $\beta o \alpha=I_{N}$. This means that $\mathrm{N}$ is a retract subact of $\mathrm{M}_{\mathrm{S}}$, since $\mathrm{N} \cong \alpha(\mathrm{N})$, so $\alpha(\mathrm{N})$ is a retract of $\mathrm{M}_{\mathrm{S}}$ and $\mathrm{f}$ is split.

The following proposition give under which condition the subacts inherit the property of C-quasi injective.

Proposition (2.5): Let $\mathrm{M}_{\mathrm{S}}$ is $\mathrm{C}$-quasi injective act. Then every fully invariant closed subact of $\mathrm{M}_{\mathrm{S}}$ is $\mathrm{C}$-quasi injective.

Proof: Let $\mathrm{N}$ be fully invariant closed subact of $\mathrm{M}_{\mathrm{S}}$ and let $\mathrm{K}$ be closed subact of $\mathrm{N}$ and let $\alpha: \mathrm{K} \rightarrow \mathrm{N}$ be $\mathrm{S}$ homomorphism. Since $N$ is closed subact of $M_{S}$, it follows that $\mathrm{K}$ is closed subact of $\mathrm{M}_{\mathrm{S}}$ by lemma (2.4) in A. shaymaa's study [14]. Then, by $\mathrm{C}$-quasi injectivity of $\mathrm{M}_{\mathrm{S}}$, there exists $\beta$ : $\mathrm{M}_{\mathrm{S}} \rightarrow \mathrm{M}_{\mathrm{S}}$ that extends $\alpha$. Since $\beta(\mathrm{N}) \subseteq \mathrm{N}$ by hypothesis, so this means there exists $\sigma$ from $\mathrm{N}$ into $\mathrm{N}$ which is the restrict of $\beta$ and extends $\alpha$. Thus $\mathrm{N}$ is $\mathrm{C}$-quasi injective.

Proposition (2.6): Every retract subact of C-M-injective act is C-M-injective act.

Proof: Assume that an S-act N is C-M-injective and A is a retract subact of S-act $N_{S}$. Let $X$ be closed subact of S-act $M_{S}$ and $\mathrm{f}$ be $\mathrm{S}$-homomorphism from $\mathrm{X}$ into $\mathrm{A}$. Since $\mathrm{N}$ is $\mathrm{C}-\mathrm{M}$ injective act, so there exists $\mathrm{S}$-homomorphism $\mathrm{g}$ from $\mathrm{M}$ into $\mathrm{N}_{S}$ such that goi ${ }_{X}=j_{A}$ of, where $j_{A}$ is the injection map of $A$ into $N_{S}$. Put $h=\pi_{A} 0 g$, where $\pi_{A}$ is the projection map of $N_{S}$ onto $A$, then hoi $_{X}=\pi_{A}$ ogoi $_{X}=\pi_{A} 0 j_{A}$ of $=f$ and $A$ is C-Minjective.

Proposition (2.7): Let $\mathrm{M}_{\mathrm{S}}$ and $\mathrm{N}_{\mathrm{S}}$ are two S-acts. If $\mathrm{N}_{\mathrm{S}}$ is $\mathrm{C}$-M-injective act, $\mathrm{B}$ is a closed subact of $\mathrm{M}_{\mathrm{S}}$, and then $\mathrm{N}_{\mathrm{S}}$ is C-B-injective act.

Proof: Let $\mathrm{X}$ be closed subact of $\mathrm{B}$, and $\mathrm{f}$ be $\mathrm{S}$ homomorphism from $X$ into $N_{S}$. Since $N_{S}$ is C-M-injective, so there exists $\mathrm{S}$-homomorphism $\mathrm{g}$ from $\mathrm{M}_{\mathrm{S}}$ into $\mathrm{N}_{\mathrm{S}}$ such that goi $i_{B} O i_{X}=f$, where $i_{X}, i_{B}$ be the inclusion map of $X$ into $B$ and $\mathrm{B}$ into $\mathrm{M}_{\mathrm{S}}$ respectively. Put $\mathrm{h}=\mathrm{goi}_{\mathrm{B}}$, then $\operatorname{hoi}_{\mathrm{X}}=\mathrm{goi}_{\mathrm{B}} \mathrm{Oi} \mathrm{i}_{\mathrm{X}}=\mathrm{f}$. Thus $\mathrm{N}_{\mathrm{S}}$ is C-B-injective act.

Corollary (2.8): Let $\mathrm{M}_{\mathrm{S}}$ and $\mathrm{N}_{\mathrm{S}}$ are two S-acts. Then, $\mathrm{N}_{\mathrm{S}}$ is $\mathrm{C}$-M-injective act if and only if $\mathrm{N}_{\mathrm{S}}$ is $\mathrm{C}$-X-injective act for every closed subact $\mathrm{X}$ of $\mathrm{M}_{\mathrm{S}}$.

Proof: Suppose that $\mathrm{N}_{\mathrm{S}}$ is C-M-injective act, by proposition (2.7), we have $\mathrm{N}_{\mathrm{S}}$ is $\mathrm{C}$-X-injective for every 
closed subact $\mathrm{X}$ of $\mathrm{M}_{\mathrm{S}}$. Conversely, since $\mathrm{M}$ is closed subact of $\mathrm{M}_{\mathrm{S}}$ and by assumption, we have $\mathrm{N}_{\mathrm{S}}$ is $\mathrm{C}-\mathrm{M}$-injective act.

Proposition (2.9): Let $\mathrm{M}_{\mathrm{S}}$ be an S-act and $\left\{\mathrm{N}_{\mathrm{i}} \mid \mathrm{i} \in \mathrm{I}\right\}$ a family of S-acts. Then $\prod_{i \in I} N_{i}$ is C-M-injective act if and only if $\mathrm{N}_{\mathrm{i}}$ is $\mathrm{C}$-M-injective act for every $\mathrm{i} \in \mathrm{I}$.

Proof: $\Rightarrow$ ) Assume that $\mathrm{N}_{\mathrm{s}}=\prod_{\mathrm{i} \in \mathrm{I}} \mathrm{N}_{\mathrm{i}}$ is C-M-injective. Let $\mathrm{X}$ is closed subact of $\mathrm{M}_{\mathrm{S}}$ and $\mathrm{f}$ is $\mathrm{S}$-homomorphism from $\mathrm{X}$ to $N_{i}$. Since $N_{S}$ is C-M-injective act then there exists $S$ homomorphism g: $\mathrm{M}_{\mathrm{S}} \rightarrow \mathrm{N}_{\mathrm{S}}$ such that goi $\mathrm{i}_{\mathrm{X}}=\mathrm{j}_{\mathrm{i}}$ of, where $\mathrm{i}_{\mathrm{X}}$ is the inclusion map of $X$ into $M_{S}$ and $j_{i}$ is the injection map of $N_{i}$ into $N_{S}$. Put $h=\pi_{i}$ og, where $\pi_{i}$ is the projection map of $N_{S}$ onto $\mathrm{N}_{\mathrm{i}}$. Then hoi $=\pi_{\mathrm{i}}$ ogoi $=\pi_{\mathrm{i}}$ oj $\mathrm{j}_{\mathrm{i}}$ of $=\mathrm{f}$.

$\Longleftarrow$ ) Assume that $\mathrm{N}_{\mathrm{i}}$ is $\mathrm{C}$-M-injective for each $\mathrm{i} \in \mathrm{I}$. Let $\mathrm{X}$ be closed subact of $\mathrm{M}_{\mathrm{S}}$ and $\mathrm{f}$ be $\mathrm{S}$-homomorphism from $\mathrm{X}$ into $N_{S}=\prod_{i \in I} N_{i}$. Since $N_{i}$ is C-M-injective act, then there exists S-homomorphism $\beta_{\mathrm{i}}: \mathrm{M}_{\mathrm{S}} \rightarrow \mathrm{N}_{\mathrm{i}}$, such that $\beta_{\mathrm{i}} \mathrm{oi}=\pi_{\mathrm{i}}$ of, so there exists S-homomorphism $\beta: \mathrm{M}_{\mathrm{S}} \rightarrow \mathrm{N}_{\mathrm{S}}$ such that $\beta=\mathrm{j}_{\mathrm{i}} \mathrm{O} \beta_{\mathrm{i}}$. We claim that $\beta$ oi $=f$. Since $\beta o \mathrm{i}=\mathrm{j}_{\mathrm{i}} \mathrm{O} \beta_{\mathrm{i}} \mathrm{Oi}=\mathrm{j}_{\mathrm{i}} \mathrm{O} \pi_{\mathrm{i}}$ of $=\mathrm{f}$, so we obtain $\mathrm{f}=\beta$ oi. Therefore $\mathrm{N}_{\mathrm{S}}$ is $\mathrm{C}$-M-injective.

Proposition (2.10): If $\mathrm{M}_{\mathrm{S}}^{\mathrm{n}}$ is $\mathrm{C}$-quasi injective act for any finite integer $n$, then $M_{S}$ is $C$-quasi injective.

Proof: Let $\mathrm{M}_{\mathrm{S}}^{\mathrm{n}}$ is $\mathrm{C}$-quasi injective act. By corollary (2.8), $M_{S}^{n}$ is C-M-quasi injective act. Since $M_{S}$ is retract of $M_{S}^{n}$, so by proposition (2.6) $\mathrm{M}_{\mathrm{S}}$ is $\mathrm{C}$-M-injective. Thus, $\mathrm{M}_{\mathrm{S}}$ is $\mathrm{C}$ quasi injective act.

Recall that an $\mathrm{S}$-acts $\mathrm{M}_{\mathrm{i}}, \mathrm{i} \in \mathrm{I}$ is called relatively $\mathrm{C}$-injective acts if $M_{i}$ is $C-M_{j}$-injective for all distinct $i, j \in I$, where $I$ is the index set.

Lemma (2.11): Let $\mathrm{M}_{1}$ and $\mathrm{M}_{2}$ be two $\mathrm{S}$-acts and $\mathrm{M}_{\mathrm{S}}=\mathrm{M}_{1} \oplus \mathrm{M}_{2}$. If $\mathrm{M}_{\mathrm{S}}$ is $\mathrm{C}$-quasi injective act, then $\mathrm{M}_{1}$ and $\mathrm{M}_{2}$ are both $\mathrm{C}$-quasi injective act and they are relatively $\mathrm{C}$ injective act.

Proof: Let $M_{s}$ be $C$-quasi injective act, this means that $M_{S}$ is C-M-injective. By proposition (2.6), we have $\mathrm{M}_{1}$ and $\mathrm{M}_{2}$ are C-M-injective acts. By corollary (2.8), we have $\mathrm{M}_{1}\left(\mathrm{M}_{2}\right)$ is $\mathrm{C}-\mathrm{M}_{2}\left(\mathrm{M}_{1}\right)$-injective act (since $\mathrm{M}_{1}$ and $\mathrm{M}_{2}$ are closed subacts).

Proposition (2.12): Every C- quasi injective act satisfies $\mathrm{C}_{2}$-condition.

Proof: Assume that $\mathrm{M}_{\mathrm{S}}$ is $\mathrm{C}$ - quasi injective act. Let $\mathrm{f}$ : $\mathrm{B} \rightarrow \mathrm{A}$ be an $\mathrm{S}$-isomorphism, where $\mathrm{A}$ and $\mathrm{B}$ are sub acts of $\mathrm{M}_{\mathrm{S}}$ and $\mathrm{A}$ is a retract of MS. Then $\mathrm{A}$ is C-M-injective act by proposition (2.6). Thus, by remarks and examples (2.2) (2), B is $\mathrm{C}-\mathrm{M}$-injective. Then, by $\mathrm{C}-\mathrm{M}$-injectivity of $\mathrm{B}$ the inclusion map $i_{B}: B \rightarrow M_{s}$ has left inverse $g: M_{S} \rightarrow B$ such that goi $i_{B}=I_{B}$. Hence by proposition (2.4), $\mathrm{i}_{\mathrm{B}}$ is splits and then $\mathrm{B}$ is a retract subact of $\mathrm{M}_{\mathrm{S}}$. Thus, $\mathrm{M}_{\mathrm{S}}$ satisfies $\mathrm{C}_{2}$-condition.

Now, we will study the relationship among C-M-injective act and injective act, extending act, continuous act.

Proposition (2.13): An S-act $\mathrm{M}_{\mathrm{S}}$ is extending act (for short $\mathrm{CS}$ act) if and only if every $\mathrm{S}$-act is $\mathrm{C}$-M-injective.

Proof: $\Rightarrow$ ) It is obvious.

$\Longleftarrow)$ Let $\mathrm{N}$ be a closed subact of S-act $\mathrm{M}_{\mathrm{S}}$. By hypothesis $\mathrm{N}$ is $\mathrm{C}$-M-injective, so by proposition (2.4), $\mathrm{N}$ is a retract subact of $\mathrm{M}_{\mathrm{S}}$. It follows that $\mathrm{M}_{\mathrm{S}}$ is CS-act.

Proposition (2.14): If every S- act is C-M-injective, then it is continuous.

Proof: Let $\mathrm{M}_{\mathrm{S}}$ be $\mathrm{S}$-act so by hypothesis $\mathrm{M}_{\mathrm{S}}$ is C-Minjective act. By proposition (2.13), an S-act $\mathrm{M}_{\mathrm{S}}$ satisfies $\mathrm{C}_{1^{-}}$ condition and by proposition (2.12), $\mathrm{M}_{\mathrm{S}}$ satisfies $\mathrm{C}_{2^{-}}$ condition. Thus $\mathrm{M}_{\mathrm{S}}$ is continuous act.

Recall that an S-act $M_{S}$ is Noetherian if every subact of $M_{S}$ is finitely generated. A monoid $\mathrm{S}$ is a right Noetherian if $\mathrm{S}_{\mathrm{S}}$ is Noetherian. Equivalently, $\mathrm{S}$ is a right Noetherian if and only if $\mathrm{S}$ satisfies the ascending chain condition for right ideals (definition1.1.30) in book of M. Kilp, U. Knauer, and A. V. Mikhalev [4, p. 21].

Before the next theorem which is a generalization of theorem (1.1) in A. K. Tiwary, S. A. Paramhans, and B. M. Pandeya' study [15], we need the following theorem:

Theorem (2.15): [11] For a monoid $\mathrm{S}$ with zero, the following conditions are equivalent:

(1) Each direct sum of injective S-acts is injective.

(2) Each direct sum of weakly injective S-acts is weakly injective.

(3) Each injective S-act is countably $\Sigma$-injective.

(4) Each finitely injective S-act is weakly injective.

(5) $\mathrm{S}$ is Noetherian.

Theorem (2.16): The following conditions are equivalent for an $\mathrm{S}-$ act $\mathrm{M}_{\mathrm{S}}$, where $\mathrm{S}$ is Noetherian monoid:

(1) The direct sum of every two C-quasi injective S-acts are $\mathrm{C}$-quasi injective acts.

(2) Every C-quasi injective act is injective.

Proof: $(1 \Rightarrow 2)$ Assume that $\mathrm{M}_{\mathrm{S}}$ is $\mathrm{C}$-quasi injective act and $\mathrm{E}(\mathrm{M})$ is injective envelope of $\mathrm{M}_{\mathrm{S}}$. Then, by assumption $\mathrm{N}_{\mathrm{S}}=\mathrm{M}_{\mathrm{S}} \oplus \mathrm{E}(\mathrm{M})$ is $\mathrm{C}$-quasi injective. Consider the injection maps i: $M_{S} \rightarrow E(M), j_{1}: E(M) \rightarrow M_{S} \oplus E(M), j_{2}: M_{S} \rightarrow M_{S} \oplus E$ (M) and $I_{M}: M_{S} \rightarrow M_{S}$ is the identity map of $M_{S}$. Let $\pi_{M}$ : $\mathrm{M}_{\mathrm{S}} \oplus \mathrm{E}(\mathrm{M}) \rightarrow \mathrm{M}_{\mathrm{S}}$ be the projection map such that $\pi_{\mathrm{M}} \mathrm{oj}_{2}=\mathrm{I}_{\mathrm{M}}$. Now, $M_{S} \oplus E(M)$ is C-quasi injective, so this implies there exists S-homomorphism g: $\mathrm{MS} \oplus \mathrm{E}(\mathrm{M}) \rightarrow \mathrm{M}_{\mathrm{S}} \oplus \mathrm{E}(\mathrm{M})$ such that $\operatorname{goj}_{1} \mathrm{oi}_{2} \mathrm{j}_{2} \mathrm{OI}_{\mathrm{M}}$, then $\pi_{\mathrm{M}}$ ogoj $_{1} \mathrm{oi}=\pi_{\mathrm{M}} \quad \mathrm{oj}_{2} \mathrm{OI}_{\mathrm{M}}$. Thus $I_{M}=\pi_{M} \operatorname{ogoj}_{1}$ oi. Put $f=\pi_{M}$ ogoj $_{1}$, then $I_{M}=$ foi. Therefore $M_{S}$ is a retract subact of $E(M)$ and then it is injective.

$(2 \Rightarrow 1)$ Let $\mathrm{M}_{\mathrm{S}}$ and $\mathrm{N}_{\mathrm{S}}$ be two $\mathrm{C}$-quasi injective S-act. By (2) $M_{S}$ and $N_{S}$ are injective which implies that the direct sum of any two injective $\mathrm{S}$-acts is injective whence $\mathrm{S}$ is Noetherian monoid by theorem (2.15) and then every injective is $\mathrm{C}$-quasi injective. Therefore, the direct sum of two $\mathrm{C}$-quasi injective is $\mathrm{C}$-quasi injective.

It is clear that every co-Hopfian is directly finite, but the converse is not true in general (for this, assume that $\mathrm{M}_{\mathrm{S}}$ is coHopfian and $f, g \in T=$ End $\left(M_{S}\right)$ such that fog $=I$, then $g$ is injective homomorphism. Since $\mathrm{M}_{\mathrm{s}}$ is co-Hopfian, so $\mathrm{g}$ is isomorphism and thus there exists $\mathrm{g}^{-1}$. Then, $\mathrm{f}=\mathrm{fgg}^{-1}=\mathrm{Ig}^{-1}=\mathrm{g}^{-1}$, so $\mathrm{gf}=\mathrm{gg}^{-1}=\mathrm{I}$ which implies that $\mathrm{M}_{\mathrm{S}}$ is directly finite). In the following proposition, we give a condition to be the converse is true:

Proposition (2.17): Every C- quasi injective act and directly finite is co-Hopfian.

Proof: Let $f$ be an injective endomorphism of $M_{S}$ and $I_{M}$ is an identity homomorphism from $M_{S}$ to $M_{S}$. Since $M_{S}$ is $\mathrm{C}-\mathrm{M}$-injective act, so there exists a homomorphism $\mathrm{g}$ : $\mathrm{M}_{\mathrm{S}} \rightarrow \mathrm{M}_{\mathrm{S}}$ such that gof $=\mathrm{I}$, since $\mathrm{M}_{\mathrm{S}}$ is directly finite, so fog $=\mathrm{I}$ which implies that $\mathrm{f}$ is onto. Hence $\mathrm{M}_{\mathrm{S}}$ is coHopfian.

The following proposition shows that the concepts of Hopfian, co-Hopfian and directly finite are coincide under C- 
quasi injectivity condition:

Proposition (2.18): Let $\mathrm{M}_{\mathrm{S}}$ is $\mathrm{C}$ - quasi injective act. Then the following concepts are equivalent:

(1) $M_{S}$ is Hopfian,

(2) $M_{S}$ is co-Hopfian,

(3) $M_{S}$ is directly finite.

Proof: $(1 \rightarrow 2)$ as every Hopfian is directly finite (For this if for any $\alpha, \beta \in$ End $\left(M_{S}\right)$ and $\alpha o \beta=I$, then this means that $\alpha$ is surjective. Since $M_{S}$ is Hopfian act and then $\alpha$ is isomorphism and $\beta$ is inverse of $\alpha$. Thus $\beta o \alpha=I$ which implies that $\mathrm{M}_{\mathrm{S}}$ is directly finite act), so by proposition (2.17), $\mathrm{M}_{\mathrm{S}}$ is co-Hopfian.

( $2 \leftrightarrow 3$ ) By proposition (2.17).

$(3 \rightarrow 1)$ Let $f$ be surjective endomorphism of $\mathrm{M}_{\mathrm{S}}$, then the inclusion map i: $\mathrm{f}(\mathrm{M}) \rightarrow \mathrm{M}_{\mathrm{S}}$ is isomorphism (since by proposition (2.17), $\mathrm{M}_{\mathrm{S}}$ is co-Hopfian). Thus foi $\mathrm{I}_{\mathrm{f}(\mathrm{M})}$. Again since $M_{S}$ is directly finite, so iof $=I_{M}$ (since $\left.f(M) \cong M_{S}\right)$. Thus $\mathrm{f}$ is injective and then it is isomorphism. Therefore, $\mathrm{M}_{\mathrm{S}}$ is Hopfian.

\section{Discussion}

In this section, we clarify what's the meaning of the results which were obtained in this article. One of these results, it is proposition (2.4) where it was demonstrated that every monomorphism from closed subact into S-act is split when the subact is C-M-injective. As for proposition (2.5), it was clarified that closed subacts of $\mathrm{C}$-quasi injective is $\mathrm{C}$-quasi injective if they are fully invariant, while, proposition (2.6) explained that subacts of $\mathrm{C}-\mathrm{M}$-injective is $\mathrm{C}-\mathrm{M}$-injective if they are retracted. In addition, we were proved in proposition (2.9) that a finite product of C-M-injective acts is C-Minjective and the converse is true also, this means if the product is C-M-injective, then each $\mathrm{M}_{\mathrm{i}}$ is C-M-injective. Besides, in proposition (2.10), we elucidated if the finite direct product is C-quasi injective, then each $\mathrm{M}_{\mathrm{i}}$ will be $\mathrm{C}$ quasi injective. Lemma (2.11) explained interesting result when $\mathrm{S}$-act $\mathrm{M}_{\mathrm{S}}$ has the form $\mathrm{M}_{\mathrm{S}}=\mathrm{M}_{1} \oplus \mathrm{M}_{2}$ and it is C-quasi injective then each of the $\mathrm{M}_{\mathrm{i}}(\mathrm{i}=1,2)$ will be $\mathrm{C}$-quasi injective and relatively $\mathrm{C}$-injective. Proposition (2.13) gave the coincide between extending act (CS-act), C-Minjective if every S-act is C-M-injective, while from proposition (2.14), we obtained that if every S-act is C-Minjective, then it will be a continuous act. Also, the theorem (2.16) showed essential identical between two important conditions which were a. direct sum of every two $\mathrm{C}$-quasi injective $\mathrm{S}$-acts is $\mathrm{C}$-quasi injective acts $\mathrm{b}$. every $\mathrm{C}$-quasi injective act is injective, when a monoid $\mathrm{S}$ is Noetherian. Furthermore, proposition (2.17) clarified that every C-quasi injective act coincides with Co-Hopfian if one can add the directly finite condition to C-quasi injective act to be Co-Hopfian, while the proposition (2.18) was demonstrated that the concepts of Hopfian, CoHopfian and directly finite will coincide if the S-act is Cquasi injective.

\section{Conclusions}

From previous theorem, examples, remark, and propositions, we can pick out some senior points as follows: We obtained an interesting result in proposition (2.4) which was: a closed subact $\mathrm{N}$ of $\mathrm{S}$-act $\mathrm{M}_{\mathrm{S}}$ is a retract subact of $\mathrm{M}_{\mathrm{S}}$ if $\mathrm{N}$ is C-M-injective. Also, proposition (2.5) gave the answer to the question raised early in the abstract which was: what are the conditions to be met in the subacts in order to inherit the property of C-quasi injectivity? In the proposition (2.6), we concluded the characterizations of C-M-injective act. From proposition (2.9) and proposition (2.10), we deduced that the direct sum of C-M-injective act is also C-M-injective and if $\mathrm{M}_{\mathrm{S}}^{\mathrm{n}}$ is $\mathrm{C}$-quasi injective act for any finite integer $\mathrm{n}$, then $\mathrm{M}_{\mathrm{S}}$ is $\mathrm{C}$-quasi injective respectively. As for the lemma (2.11), we got that if $M_{S}=M_{1} \oplus M_{2}$ is C-quasi injective acts, then $\left.\mathrm{M}_{\mathrm{i}}(\mathrm{i}=1,2)\right)$ are relatively $\mathrm{C}$-injective acts.

In addition, proposition (2.13) and proposition (2.14) gave the relationship among C-M-injective acts with CS-acts and continuous acts respectively where these propositions illustrate that if every S-act is C-M-injective, then it will be a CS and continuous act respectively. In the theorem (2.16), we elucidated important result which is the existence of Noetherian monoid was solved the problem of the identical between the following conditions, I. The direct sum of two $\mathrm{C}$-quasi injective $\mathrm{S}$-acts is $\mathrm{C}$-quasi injective acts and II. Every C-quasi injective act is injective. Finally, the relationship among the notions, C-quasi injective, Hopfian, co-Hopfian, and directly finite was illustrated in proposition (2.17) and proposition (2.18).

\section{Acknowledgements}

At first, the authors would like to present with their work to the spirit of their fathers. Besides, the authors would like to thank their families for their support for the completion of this work. The authors thank the editor and anonymous reviewers for their useful comments and suggestions. Also, the authors would like to thank Mustansiriyah University (www.uomustansiriyah.edu.iq) Baghdad-Iraq for its support in the present work.

\section{References}

[1] M. Kilp, U. Knauer, \& A. V. Mikhalev, "Monoids acts and categories with applications to wreath products and graphs", a handbook for students and researchers, Berlin, W. de Gruyter, (2000). Retrieved from https://www.amazon.com/CategoriesApplications-Expositions-MathematicsDegruyter/dp/3110152487

[2] A. Shaymaa, "About the generalizations in systems over monoids", LAP LAMBERT Academic Publishing, Germany, (2018). Retrieved from https://www.amazon.com/Aboutgeneralizations-systems-over-monoids/dp/6135841955

[3] A. Shaymaa, "Generalizations of quasi injective acts over monoids", PhD. thesis, College of Science, University of Al-Mustansiriyah, Baghdad, Iraq, (2015). Retrieved from https://www.academia.edu/35100206/GENERALIZATION $S$ OF QUASI INJECTIVE SSYYSTEMS OVVER MONOIDS 
[4] A. Shaymaa, "Pseudo C-M-injective and pseudo C-quasi principally injective acts over monoids", Journal of Progressive Research in Mathematics (JPRM), 6 (3), pp. 788802, (2016). Retrieved from http://scitecresearch.com/journals/index.php/jprm/article/view 1580

[5] A. Shaymaa, "Pseudo IC-injective systems over monoids", Global Journal of mathematics (GJM), 6 (3), pp. 636-642, (2016). Retrieved from https://www.researchgate.net/publication/324835936_PSEUD O_IC-INJECTIVE_SYSTEMS_OVER_MONOIDS

[6] Berthiaume P., "The injective envelope of S-sets", Canad. Math. Bull., 10 (2), pp. 261-272, (1967). Retrieved from https://www.cambridge.org/core/services/aop-cambridgecore/content/view/CC5C688B45202403E48AE8B5C75CEC0 F/S0008439500067242a.pdf/injective_envelope_of_ssets.pdf

[7] Shaymaa A., "On Finitely Generated in S-systems over monoids", Noor Publishing, Germany, (2018). Retrieved from https://www.amazon.com/s?k=On+Finitely+Generated+in+Ssystems + over+Monoids\&ref=nb_sb_noss

[8] T.Yan, "Generalized injective S-acts on a monoid", Advances in mathematics, 40 (4), pp. 421-432, (2011). Retrieved from http://sci.upc.edu.cn/2016/0926/c6900a96616/page.htm

[9] K. Sungjin and K. Jupil, "Weakly large subacts of S-act", J. of Chungcheong Math. Soc., 20 (4), pp486-493, (2007).
[10] C. V. Hinkle and Jr., "The extended centralizer of an S-set", Pacific journal of mathematics, 53 (1), pp163-170, (1974). Retrieved from https://msp.org/pjm/1974/53-1/pjm-v53-n1p14-s.pdf

[11] J. Ahsan, "Monoids characterized by their quasi injective S-acts", Semigroup forum, 36 (3), pp 285-292, (1987). Retrieved from https://link.springer.com/article/10.1007\%2FBF02575022

[12] A. M. Lopez, Jr. and J. K. Luedeman, "Quasi-injective S-acts and their S-endomorphism Semigroup", Czechoslovak Math. J., 29 (104), pp97-104, (1979). Retrieved from https://dml.cz/bitstream/handle/10338.dmlcz/101581/CzechM athJ_29-1979-1_11.pdf

[13] M. S. Abbas and A. Shaymaa, "Principally quasi injective act over monoid", Journal of advances in mathematics, 10 (1), 3152-3162, (2015). Retrieved from https://www.researchgate.net/publication/324845514_PRINCI PALLY_QUASI_INJECTIVE_SYSTEM_OVER_MONOID

[14] A. Shaymaa, "Extending and P-extending S-act over Monoids", International Journal of Advanced Scientific and Technical Research, 2 (7), pp. 171-178, (2017). Retrieved from https://www.academia.edu/34905983/Extending_and_Pextending_S-act_over_Monoids

[15] A. K. Tiwary, S. A. Paramhans, and B. M. Pandeya, "Generalization of quasi injectivity", Progress of Mathematics, 13 (1-2), pp. 31-40, (1979). 